\title{
APPROXIMATE EIGENVALUE DECOMPOSITION OF PARA-HERMITIAN SYSTEMS THROUGH SUCCESSIVE FIR PARAUNITARY TRANSFORMATIONS
}

\author{
Andre Tkacenko \\ Signal Processing Research Group \\ Jet Propulsion Laboratory \\ California Institute of Technology \\ 4800 Oak Grove Drive, Pasadena, CA 91109 \\ E-mail: Andre.Tkacenko@jpl.nasa.gov
}

\begin{abstract}
The eigenvalue decomposition (EVD) of a Hermitian matrix in terms of unitary matrices is well known. In this paper, we present an algorithm for the approximate EVD (AEVD) of a para-Hermitian $(\mathrm{PH})$ system. Here, the approximate diagonalization is carried out successively by applying degree-1 finite impulse response (FIR) paraunitary (PU) transformations. The system parameters are chosen to make the zeroth order diagonal energy (ZODE) nondecreasing at each stage. Simulation results presented for the design of a signal-adapted PU filter bank (FB) show close agreement with the behavior of the infinite order principal component FB (PCFB).
\end{abstract}

Index Terms - matrix decomposition, approximation methods, polynomial approximation

\section{INTRODUCTION}

A well known result in matrix theory is the eigenvalue decomposition (EVD) of a Hermitian matrix in terms of unitary matrices [1]. Specifically, if $\mathbf{A}$ denotes a $p \times p$ Hermitian matrix (i.e., $\mathbf{A}^{\dagger}=\mathbf{A}$ ), then $\mathbf{A}$ can be expressed as follows [1].

$$
\mathbf{A}=\mathbf{U} \boldsymbol{\Lambda} \mathbf{U}^{\dagger}
$$

Here, $\mathbf{U}$ is a $p \times p$ matrix of eigenvectors of $\mathbf{A}$ that is unitary (i.e., $\mathbf{U}^{\dagger} \mathbf{U}=\mathbf{I}_{p}$ ), while $\boldsymbol{\Lambda}$ is a $p \times p$ diagonal matrix of eigenvalues of $\mathbf{A}$. A method to compute this EVD is given in [1].

With the advent of multiple input multiple output (MIMO) theory [2] has come interest in para-Hermitian (PH) systems $[3,4]$. An $M \times M$ MIMO transfer function $\mathbf{A}(z)$ with impulse response $\mathbf{a}[n]$ is said to be $\mathrm{PH}$ iff $\mathbf{A}^{\dagger}\left(1 / z^{*}\right)=\mathbf{A}(z)$ for all $z$ or equivalently $\mathbf{a}^{\dagger}[-n]=\mathbf{a}[n]$ for all $n$. If $z=e^{j 2 \pi f}$ for $0 \leq f<1$ is contained in the region of convergence (ROC) [5] of $\mathbf{A}(z)$, then $\mathbf{A}(z)$ is $\mathrm{PH}$ iff $\mathbf{A}^{\dagger}\left(e^{j 2 \pi f}\right)=\mathbf{A}\left(e^{j 2 \pi f}\right)$ for all $0 \leq f<1$, meaning that $\mathbf{A}\left(e^{j 2 \pi f}\right)$ is Hermitian for all $f$.

The research described in this publication was carried out at the Jet Propulsion Laboratory, California Institute of Technology, under a contract with the National Aeronautics and Space Administration.
Assuming $z=e^{j 2 \pi f}$ is in the ROC of $\mathbf{A}(z)$, then an EVD for $\mathbf{A}\left(e^{j 2 \pi f}\right)$ can be computed in theory as follows from (1).

$$
\mathbf{A}\left(e^{j 2 \pi f}\right)=\mathbf{U}\left(e^{j 2 \pi f}\right) \boldsymbol{\Lambda}\left(e^{j 2 \pi f}\right) \mathbf{U}^{\dagger}\left(e^{j 2 \pi f}\right) \quad \forall f
$$

Here, $\mathbf{U}\left(e^{j 2 \pi f}\right)$ is an $M \times M$ matrix of eigenvectors of $\mathbf{A}\left(e^{j 2 \pi f}\right)$ that is paraunitary (PU) (i.e., $\mathbf{U}^{\dagger}\left(e^{j 2 \pi f}\right) \mathbf{U}\left(e^{j 2 \pi f}\right)=$ $\mathbf{I}_{M}$ for all $\left.f[2]\right)$, while $\boldsymbol{\Lambda}\left(e^{j 2 \pi f}\right)$ is an $M \times M$ diagonal matrix of eigenvalues of $\mathbf{A}\left(e^{j 2 \pi f}\right)$. Since (2) must hold for all $f$, the EVD in (2) is said to be pointwise in frequency [6].

The problem with computing an exact EVD of a PH system as in (2) is that in general, the PU system $\mathbf{U}\left(e^{j 2 \pi f}\right)$ may not be realizable [6]. More emphasis has thus been on obtaining an approximate EVD (AEVD) using realizable PU functions, such as finite impulse response (FIR) PU systems [3, 4].

Here, we present an AEVD algorithm for PH systems via successive degree-1 FIR PU transformations. We show how to choose the parameters of such a FIR PU system to make the zeroth order diagonal energy (ZODE) of the resultant $\mathrm{PH}$ system nondecreasing. As more transformations are applied, the PH system approximately becomes more diagonal.

This algorithm is similar to the sequential best rotation (SBR2) method [3], but with two main differences. As our aim is to maximize the ZODE, the entire impulse response of the PH system need not be known. The algorithm can thus run in the time or frequency domain, unlike SBR2 which must operate in the time domain. Also, at each stage of our algorithm, the FIR PU system degree increases by 1 . This is in contrast to SBR2, which has a variable degree increase at each step.

\subsection{Outline}

We review FIR PU transfer functions in Sec. 2, where we introduce Vaidyanathan's canonical decomposition of such systems [2]. In Sec. 3, we discuss the different roles that the parameters of a degree-1 FIR PU transformation of a PH system have on increasing the ZODE of the resultant system. We present our successive algorithm for AEVD of $\mathrm{PH}$ systems in Sec. 4 . The parameter choices selected to increase the ZODE 
are detailed in Sec. 4.1 and 4.2. Simulation results for signaladapted PU filter bank (FB) design are given in Sec. 5, where we show the close agreement between the FIR PU FBs generated and the infinite order principal component FB (PCFB) [6]. Concluding remarks are made in Sec. 6.

\subsection{Notations}

All notations are as in $[5,2]$. The $(k, \ell)$-th element of any $p \times r$ matrix $\mathbf{A}$ will be denoted $[\mathbf{A}]_{k, \ell}$. For any $p \times r$ transfer function $\mathbf{A}(z)$, we will use the tilde notation to denote its $r \times p$ paraconjugate [2], i.e., $\widetilde{\mathbf{A}}(z) \triangleq \mathbf{A}^{\dagger}\left(1 / z^{*}\right)$. Also, for any $p \times r$ system $\mathbf{A}(z)$ with impulse response $\mathbf{a}[n]$, we define the ZODE of $\mathbf{A}(z)$, denoted by $\zeta_{\mathbf{A}}$, to be the energy of the diagonal components of the zeroth order term $\mathbf{a}[0]$, given by,

$$
\zeta_{\mathbf{A}} \triangleq \sum_{m=0}^{\min (p, r)-1}\left|[\mathbf{a}[0]]_{m, m}\right|^{2}
$$

Finally, for any $p \times r$ matrix $\mathbf{A}$, we will denote by $\|\mathbf{A}\|_{F}$ the Frobenius norm [1] of $\mathbf{A}$ given by $\|\mathbf{A}\|_{F}=\sqrt{\operatorname{Tr}\left[\mathbf{A}^{\dagger} \mathbf{A}\right]}$.

\section{REVIEW OF FIR PU SYSTEMS}

Let $\mathbf{F}(z)$ denote a $p \times r$ causal FIR PU system of McMillan degree $N$ [2]. We assume $p \geq r$ in order to satisfy the PU condition $\widetilde{\mathbf{F}}(z) \mathbf{F}(z)=\mathbf{I}_{r}$. In [2], Vaidyanathan showed that any such $\mathbf{F}(z)$ could be completely parameterized in terms of Householder-like [1] degree-1 building blocks. Specifically, $\mathbf{F}(z)$ is a causal degree- $N$ FIR PU system iff it is of the form,

$$
\mathbf{F}(z)=\left(\prod_{k=N}^{1} \mathbf{V}_{k}(z)\right) \mathbf{U}
$$

where $\mathbf{V}_{k}(z)$ is a degree-1 building block given by,

$$
\mathbf{V}_{k}(z)=\mathbf{I}_{p}-\mathbf{v}_{k} \mathbf{v}_{k}^{\dagger}+z^{-1} \mathbf{v}_{k} \mathbf{v}_{k}^{\dagger}, 1 \leq k \leq N
$$

Here, the $p \times 1$ vectors $\mathbf{v}_{k}$ are unit norm vectors (i.e., $\mathbf{v}_{k}^{\dagger} \mathbf{v}_{k}=$ 1 ) for all $k$ and $\mathbf{U}$ is a $p \times r$ unitary matrix (i.e., $\mathbf{U}^{\dagger} \mathbf{U}=$ $\mathbf{I}_{r}$ ). In the special case $N=0$, the product appearing in (4) degenerates to an empty value of $\mathbf{I}_{p}$ and so $\mathbf{F}(z)=\mathbf{U}$.

\section{USING A DEGREE-1 FIR PU TRANSFORMATION TO INCREASE THE ZODE OF A PH SYSTEM}

For the degree- 1 case of $p=r=M$, we get, using (5) in (4),

$$
\mathbf{F}(z)=\underbrace{\left(\mathbf{I}-\mathbf{v} \mathbf{v}^{\dagger}+z^{-1} \mathbf{v} \mathbf{v}^{\dagger}\right)}_{\mathbf{V}(z)} \mathbf{U}
$$

where $\mathbf{I} \triangleq \mathbf{I}_{M}$ and $\mathbf{v} \triangleq \mathbf{v}_{1}$. Let $\mathbf{A}(z)$ be some $M \times M \mathrm{PH}$ system and consider the degree-1 FIR PU transformation,

$$
\mathbf{Y}(z) \triangleq \widetilde{\mathbf{F}}(z) \mathbf{A}(z) \mathbf{F}(z)=\mathbf{U}^{\dagger} \underbrace{(\tilde{\mathbf{V}}(z) \mathbf{A}(z) \mathbf{V}(z))}_{\mathbf{X}(z)} \mathbf{U}
$$

The key to choosing $\mathbf{v}$ and $\mathbf{U}$ from (6) to ensure that the ZODE of transformed $\mathrm{PH}$ system $\mathbf{Y}(z)$ is greater than or equal to that of the original $\mathbf{A}(z)$ lies in recognizing the different roles that $\mathbf{v}$ and $\mathbf{U}$ have for accomplishing this goal.

From (6), we see that $\mathbf{v}$ governs memory effects in (7), whereas $\mathbf{U}$ only has a memoryless impact. Heuristically, this implies that $\mathbf{v}$ can temporally move energy, whereas $\mathbf{U}$ can spatially allocate it. Hence, to increase the ZODE, we opt to pick $\mathbf{v}$ to collect energy in the zeroth order term, and then choose $\mathbf{U}$ to distribute it along the diagonal elements.

Quantitatively, if $\mathbf{a}[0], \mathbf{x}[0]$, and $\mathbf{y}[0]$ denote, respectively, the zeroth order terms of $\mathbf{A}(z), \mathbf{X}(z)$, and $\mathbf{Y}(z)$ from (7), then $\mathbf{v}$ and $\mathbf{U}$ are chosen as follows. First, $\mathbf{v}$ is selected to collect the energy of $\mathbf{A}(z)$ so that the following condition holds.

$$
\|\mathbf{x}[0]\|_{F}^{2} \geq\|\mathbf{a}[0]\|_{F}^{2} \geq \zeta_{\mathbf{A}}
$$

Note that the second inequality in (8) is always satisfied by exploiting the ZODE definition from (3). Once $\mathbf{v}$ has been set, $\mathbf{U}$ is chosen to distribute the energy of $\mathbf{x}[0]$ along the diagonal components. Namely, as $\mathbf{x}[0]$ is Hermitian, we select $\mathbf{U}$ such that $\mathbf{y}[0]=\mathbf{U}^{\dagger} \mathbf{x}[0] \mathbf{U}$ is diagonal, which by (1), is possible. This ensures that $\zeta_{\mathbf{Y}}=\|\mathbf{x}[0]\|_{F}^{2}$, and so by (8), we have,

$$
\zeta_{\mathbf{Y}} \geq \zeta_{\mathbf{A}}
$$

and thus the ZODE of the transformed system $\mathbf{Y}(z)$ from (7) is greater than or equal to that of the original $\mathrm{PH}$ system $\mathbf{A}(z)$.

To see why $\mathbf{Y}(z)$ is heuristically more diagonal than $\mathbf{A}(z)$, note that they have the same energy. That is, if $\mathcal{E}_{\mathbf{H}}$ is the energy of a system $\mathbf{H}(z)$ with impulse response $\mathbf{h}[n]$,

$$
\mathcal{E}_{\mathbf{H}} \triangleq \int_{0}^{1}\left\|\mathbf{H}\left(e^{j 2 \pi f}\right)\right\|_{F}^{2} d f=\sum_{n \in \mathbb{Z}}\|\mathbf{h}[n]\|_{F}^{2}
$$

then $\mathcal{E}_{\mathbf{Y}}=\mathcal{E}_{\mathbf{A}}$ from (7) as $\mathbf{F}(z)$ is PU. If (9) holds, then some of the off-diagonal zeroth order energy and non-zeroth order energy of $\mathbf{A}(z)$ was thus shifted to the ZODE of $\mathbf{Y}(z)$.

By applying additional degree-1 FIR PU transformations, we can further improve the diagonalization of the original $\mathrm{PH}$ system by ensuring that the ZODE of the resultant systems are nondecreasing. This forms the basis for the AEVD algorithm.

\section{ALGORITHM FOR AEVD OF PH SYSTEMS VIA SUCCESSIVE FIR PU TRANSFORMATIONS}

Let $\mathbf{A}(z)$ be any $M \times M \mathrm{PH}$ system with impulse response $\mathbf{a}[n]$ and let $N$ denote the desired degree of an FIR PU approximate diagonalizer of $\mathbf{A}(z)$. Then, do the following.

1. Compute $\mathbf{A}_{0}(z)$ as follows.

$$
\mathbf{A}_{0}(z) \triangleq \mathbf{U}_{0}^{\dagger} \mathbf{A}(z) \mathbf{U}_{0}=\sum_{n \in \mathbb{Z}} \mathbf{a}_{0}[n] z^{-n}
$$

Here, $\mathbf{U}_{0}$ is calculated as described in Sec. 4.2, i.e., $\mathbf{U}_{0}$ diagonalizes $\mathbf{a}[0]$ and arranges the eigenvalues in descending order. Set the index variable $k$ to 1 . 
2. If $k=N+1$, stop as we are done. Otherwise, define $\mathbf{V}_{k}(z), \mathbf{B}_{k}(z)$, and $\mathbf{A}_{k}(z)$ as follows.

$$
\begin{aligned}
& \mathbf{V}_{k}(z) \triangleq \mathbf{I}-\mathbf{v}_{k} \mathbf{v}_{k}^{\dagger}+z^{-1} \mathbf{v}_{k} \mathbf{v}_{k}^{\dagger} \\
& \mathbf{B}_{k}(z) \triangleq \widetilde{\mathbf{V}}_{k}(z) \mathbf{A}_{k-1}(z) \mathbf{V}_{k}(z)=\sum_{n \in \mathbb{Z}} \mathbf{b}_{k}[n] z^{-n} \\
& \mathbf{A}_{k}(z) \triangleq \mathbf{U}_{k}^{\dagger} \mathbf{B}_{k}(z) \mathbf{U}_{k}=\sum_{n \in \mathbb{Z}} \mathbf{a}_{k}[n] z^{-n}
\end{aligned}
$$

Here, $\mathbf{v}_{k}$ is computed from (16) in Sec. 4.1, while $\mathbf{U}_{k}$ is calculated as described in Sec. 4.2, i.e., $\mathbf{U}_{k}$ diagonalizes $\mathbf{b}_{k}[0]$ and arranges the eigenvalues in descending order.

3. Update the index variable $k$ to $k \rightarrow k+1$ and go to 2 .

At the end of the AEVD algorithm, $\mathbf{A}(z)$ is approximately diagonalized to $\mathbf{A}_{N}(z)$, which is given by the following.

$$
\mathbf{A}_{N}(z)=\widetilde{\mathbf{F}}_{N}(z) \mathbf{A}(z) \mathbf{F}_{N}(z)
$$

Here, $\mathbf{F}_{N}(z)$ is an FIR PU system of degree $N$ as follows.

$$
\mathbf{F}_{N}(z)=\mathbf{U}_{0}\left(\prod_{k=1}^{N} \mathbf{V}_{k}(z) \mathbf{U}_{k}\right)
$$

\subsection{Selected Choice of $\mathbf{v}_{k}$}

As motivated in Sec. 3 via (8), the role of $\mathbf{v}_{k}$ in (11)-(12) is to collect as much energy as possible into the zeroth order term $\mathbf{b}_{k}[0]$. This is done by maximizing $\left\|\mathbf{b}_{k}[0]\right\|_{F}^{2}$. Combining (11) into (12), we have, after some algebraic manipulation,

$$
\left\|\mathbf{b}_{k}[0]\right\|_{F}^{2}=\left\|\mathbf{a}_{k-1}[0]\right\|_{F}^{2}+2 \xi_{k}\left(\mathbf{v}_{k}\right)
$$

Here, $\xi_{k}\left(\mathbf{v}_{k}\right)$ is the quartic objective function,

$$
\xi_{k}\left(\mathbf{v}_{k}\right) \triangleq \mathbf{v}_{k}^{\dagger}\left[\mathbf{C}_{k}\left(\mathbf{I}-\mathbf{v}_{k} \mathbf{v}_{k}^{\dagger}\right) \mathbf{C}_{k}^{\dagger}-\mathbf{D}_{k}\left(\mathbf{I}-\mathbf{v}_{k} \mathbf{v}_{k}^{\dagger}\right) \mathbf{D}_{k}\right] \mathbf{v}_{k}
$$

and $\mathbf{C}_{k} \triangleq \mathbf{a}_{k-1}[1]$ and $\mathbf{D}_{k} \triangleq \mathbf{a}_{k-1}[0]$. Note that because of the choice of $\mathbf{U}_{k-1}$ (see Steps 1 and 2 of the above algorithm and Sec. 4.2), $\mathbf{a}_{k-1}[0]=\mathbf{D}_{k}$ is a diagonal Hermitian matrix.

Ideally, $\left\|\mathbf{b}_{k}[0]\right\|_{F}^{2}$ from (14) or equivalently $\xi_{k}\left(\mathbf{v}_{k}\right)$ from (15) should be maximized subject to the quadratic constraint $\mathbf{v}_{k}^{\dagger} \mathbf{v}_{k}=1$. Unfortunately, there does not appear to be a closed form solution to this optimization problem. Instead, we opt to maximize $\xi_{k}\left(\mathbf{v}_{k}\right)$ from among the set of eigenvectors of $\mathbf{D}_{k}$, which is the set of unit element vectors $\left\{\mathbf{e}_{\ell}\right\}$ [1]. Any such choice ensures that $\xi_{k}\left(\mathbf{v}_{k}\right)$ is nonnegative since the nonpositive term involving $\mathbf{D}_{k}$ from (15) is annihilated and thus,

$$
\xi_{k}\left(\mathbf{e}_{\ell}\right)=\mathbf{e}_{\ell}^{\dagger}\left[\mathbf{C}_{k}\left(\mathbf{I}-\mathbf{e}_{\ell} \mathbf{e}_{\ell}^{\dagger}\right) \mathbf{C}_{k}^{\dagger}\right] \mathbf{e}_{\ell} \geq 0
$$

Hence, we opt to choose $\mathbf{v}_{k}$ as follows here.

$$
\mathbf{v}_{k}=\mathbf{e}_{\ell_{\mathrm{opt}}}, \ell_{\mathrm{opt}}=\underset{0 \leq \ell \leq M-1}{\arg \max } \xi_{k}\left(\mathbf{e}_{\ell}\right)
$$

\subsection{Selected Choice of $\mathbf{U}_{k}$}

As alluded to in Sec. 3, the role of $\mathbf{U}_{k}$ in (10) and (13) is to distribute the energy of the zeroth order term $\mathbf{a}_{k}[0]$ along the diagonal components. Note that $\mathbf{a}_{0}[0]=\mathbf{U}_{0}^{\dagger} \mathbf{a}[0] \mathbf{U}_{0}$ and $\mathbf{a}_{k}[0]=\mathbf{U}_{k}^{\dagger} \mathbf{b}_{k}[0] \mathbf{U}_{k}$ from (10) and (13), respectively. Also note that $\mathbf{a}[0]$ and $\mathbf{b}_{k}[0]$ for all $k$ are Hermitian. Thus, from (1), $\mathbf{a}_{k}[0]$ can be diagonal by choosing $\mathbf{U}_{k}$ to be a matrix of eigenvectors of $\mathbf{a}[0]$ for $k=0$ and $\mathbf{b}_{k}[0]$ for $1 \leq k \leq N$.

Similar to the Karhunen-Loève transform (KLT) [6], we opt to choose $\mathbf{U}_{k}$ such that the eigenvalues are arranged in $d e$ scending order. Hence, $\mathbf{U}_{k}$ is chosen so that $\mathbf{a}_{k}[0]$ is diagonal with the diagonal elements arranged in descending order.

\section{SIMULATION RESULTS}

To test our algorithm, we considered the design of a signaladapted PU FB [7]. Suppose $x[n]$ is a cyclo wide sense stationary (WSS) process of period $M$ [2] input to a uniform $M$-channel PU FB with $M \times M$ synthesis polyphase matrix $\mathbf{F}(z)$ [2]. Then, the $M$-fold blocked form of $x[n]$ [2], denoted $\mathbf{x}[n]$, is an $M \times 1$ WSS process with some power spectral density (PSD) $\mathbf{S}_{\mathbf{x x}}(z)$ [2]. The $M \times 1$ subband process $\mathbf{y}[n]$ is obtained by filtering $\mathbf{x}[n]$ with the analysis polyphase matrix $\mathbf{H}(z)=\widetilde{\mathbf{F}}(z)$ [2]. Thus, the PSD of $\mathbf{y}[n]$, denoted $\mathbf{S}_{\mathbf{y y}}(z)$, is,

$$
\mathbf{S}_{\mathbf{y y}}(z)=\widetilde{\mathbf{F}}(z) \mathbf{S}_{\mathbf{x x}}(z) \mathbf{F}(z)
$$

A PCFB is a special PU FB in which $\mathbf{S}_{\mathbf{y y}}\left(e^{j 2 \pi f}\right)$ exhibits total decorrelation and spectral majorization [6]. This means $\mathbf{F}\left(e^{j 2 \pi f}\right)$ is an EVD of $\mathbf{S}_{\mathbf{x x}}\left(e^{j 2 \pi f}\right)$ in which the diagonal of $\mathbf{S}_{\mathbf{y y}}\left(e^{j 2 \pi f}\right)$ is in descending order pointwise in frequency [6].

If $x[n]$ is itself WSS, the analysis/synthesis filters of the PCFB have ideal brick-wall responses [6]. As such filters are of infinite order, it is insightful to consider the design of a realizable PU FB formed by an AEVD of $\mathbf{S}_{\mathbf{x x}}\left(e^{j 2 \pi f}\right)$.

Suppose $x[n]$ is a real autoregressive (AR) process [5] with poles at $0.95 e^{ \pm j 2 \pi(0.1)}, 0.8 e^{ \pm j 2 \pi(0.45)}$, and that the number of channels is $M=4$. With this setup, the AEVD algorithm was run on $\mathbf{S}_{\mathbf{x x}}\left(e^{j 2 \pi f}\right)$ for various FIR PU degrees $N$.

To show the AEVD algorithm behavior relative to the PCFB, we calculated the subband variance proportion $P(L)$ from $L$ subbands, where $1 \leq L \leq M$. This is defined as [7],

$$
P(L) \triangleq \frac{\sum_{k=0}^{L-1} \sigma_{y_{k}}^{2}}{\sum_{k=0}^{M-1} \sigma_{y_{k}}^{2}}, \sigma_{y_{k}}^{2}=\int_{0}^{1}\left[\mathbf{S}_{\mathbf{y y}}\left(e^{j 2 \pi f}\right)\right]_{k, k} d f
$$

Among all PU FBs, the PCFB maximizes $P(L)$ for all $L$ [6].

A plot of $P(L)$ from (17) is shown in Fig. 1 for PU FBs designed for various $N$ along with the PCFB. As $N$ increased, the FBs designed came closer to the behavior of the PCFB.

To chart the ZODE progression, we designed an FIR PU FB with $N=100$. In Fig. 2, we plotted the evolution of the ZODE at the $k$-th step, $\zeta_{k}$, normalized by the total energy, $\mathcal{E}_{\mathbf{x}}$. The ZODE monotonically nondecreased with $k$ as expected. 


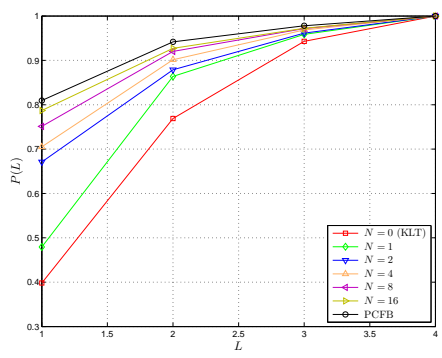

Fig. 1. Subband variance proportion $P(L)$ from (17) obtained by preserving $L$ subbands. (The case $N=0$ is the KLT [6].)

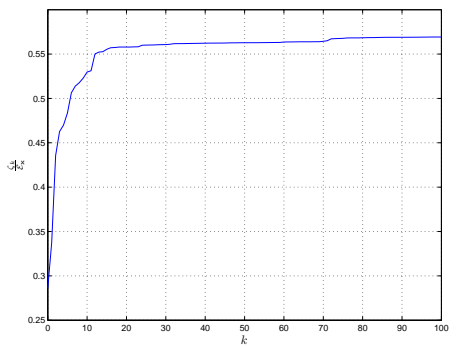

Fig. 2. Evolution of the ZODE of the AEVD transformed system at the $k$-th stage $\zeta_{k}$, normalized by the total energy $\mathcal{E}_{\mathbf{x}}$.

To assess decorrelating abilities, we measured the offdiagonal energy at the $k$-th stage. This quantity, denoted here by $\eta_{k}$, is defined as follows.

$$
\eta_{k} \triangleq \int_{0}^{1}\left\|\mathbf{S}_{\mathbf{y}_{k} \mathbf{y}_{k}}\left(e^{j 2 \pi f}\right)-\operatorname{diag}\left(\mathbf{S}_{\mathbf{y}_{k} \mathbf{y}_{k}}\left(e^{j 2 \pi f}\right)\right)\right\|_{F}^{2} d f
$$

Here, $\mathbf{S}_{\mathbf{y}_{k} \mathbf{y}_{k}}\left(e^{j 2 \pi f}\right)$ is the AEVD transformed system at the $k$-th step and $\operatorname{diag}(\mathbf{A})$ denotes a diagonal matrix formed from the diagonal components of some matrix $\mathbf{A}$.

A plot of $\eta_{k}$ from (18) normalized by $\mathcal{E}_{\mathrm{x}}$ is shown in Fig. 3 on a dB scale. The off-diagonal energy fluctuated but generally decreased. Here, $\eta_{k} / \mathcal{E}_{\mathbf{x}}$ ended at $-18.9 \mathrm{~dB}$, corresponding to an off-diagonal energy of $1.3 \%$ of the total energy. This elucidates the decorrelating aspects of the AEVD algorithm.

Finally, to show spectral majorization tendencies, we looked at the diagonal components of the transformed PSD for $N=100$. In Fig. 4, each $m$-th subband PSD $S_{y_{m} y_{m}}\left(e^{j 2 \pi f}\right)$ is shown on a dB scale for $0 \leq m \leq 3$. For most frequencies $f$, we have, $S_{y_{0} y_{0}}\left(e^{j 2 \pi f}\right) \geq S_{y_{1} y_{1}}\left(e^{j 2 \pi f}\right) \geq$ $S_{y_{2} y_{2}}\left(e^{j 2 \pi f}\right) \geq S_{y_{3} y_{3}}\left(e^{j 2 \pi f}\right)$, and so the FIR PU FB almost completely spectrally majorized the subbands [6].

\section{CONCLUDING REMARKS}

Future work consists of enhancing the AEVD algorithm by improving the choice of the vector $\mathbf{v}_{k}$ proposed in Sec. 4.1. This could be achieved, for example, by using a gradient based search technique on the objective $\xi_{k}\left(\mathbf{v}_{k}\right)$ from (15).

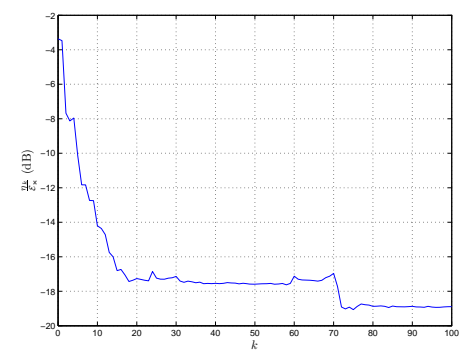

Fig. 3. Evolution of the off-diagonal energy $\eta_{k}$ from (18) of the transformed system at the $k$-th stage, normalized by $\mathcal{E}_{\mathrm{x}}$.

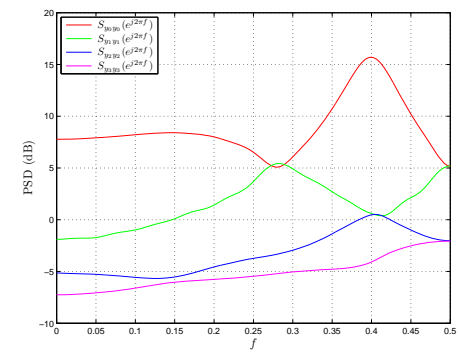

Fig. 4. Subband signal PSDs after passing through an AEVD designed FIR PU FB of degree $N=100$.

\section{REFERENCES}

[1] R. A. Horn and C. R. Johnson, Matrix Analysis. Cambridge, UK: Cambridge University Press, 1985.

[2] P. P. Vaidyanathan, Multirate Systems and Filter Banks. Englewood Cliffs, NJ: Prentice Hall PTR, 1993.

[3] J. G. McWhirter, P. D. Baxter, T. Cooper, S. Redif, and J. Foster, "An EVD algorithm for para-Hermitian polynomial matrices," IEEE Trans. Signal Process., vol. 55, no. 5, pp. 2158-2169, May 2007.

[4] M. Davies, S. Lambotharan, J. Foster, J. Chambers, and J. McWhirter, "Polynomial matrix QR decomposition and iterative decoding of frequency selective MIMO channels," in Proc. IEEE Wireless Communications \& Networking Conference (WCNC 2009), Budapest, Hungary, Apr. 5-8, 2009, pp. 1-6.

[5] A. V. Oppenheim and R. W. Schafer, Discrete-Time Signal Processing, 3rd ed. Upper Saddle River, NJ: Prentice-Hall, Inc., 2009.

[6] P. P. Vaidyanathan, "Theory of optimal orthonormal subband coders," IEEE Trans. Signal Process., vol. 46, no. 6, pp. 1528-1543, Jun. 1998.

[7] A. Tkacenko and P. P. Vaidyanathan, "Iterative greedy algorithm for solving the FIR paraunitary approximation problem," IEEE Trans. Signal Process., vol. 54, no. 1, pp. 146-160, Jan. 2006. 